\title{
Ideals and their complements in commutative semirings
}

\author{
Ivan Chajda ${ }^{1} \cdot$ Helmut Länger ${ }^{1,2}$
}

Published online: 31 August 2018

(C) The Author(s) 2018

\begin{abstract}
We study conditions under which the lattice $\mathbf{I d} \mathbf{R}$ of ideals of a given a commutative semiring $\mathbf{R}$ is complemented. At first we check when the annihilator $I^{*}$ of a given ideal $I$ of $\mathbf{R}$ is a complement of $I$. Further, we study complements of annihilator ideals. Next we investigate so-called Łukasiewicz semirings. These form a counterpart to MV-algebras which are used in quantum structures as they form an algebraic semantic of many-valued logics as well as of the logic of quantum mechanics. We describe ideals and congruence kernels of these semirings with involution. Finally, using finite unitary Boolean rings, a construction of commutative semirings with complemented lattice of ideals is presented.
\end{abstract}

Keywords Commutative semiring $\cdot$ Idempotent semiring $\cdot$ Ideal $\cdot$ Annihilator $\cdot$ Lattice of ideals $\cdot$ Complement $\cdot$ Łukasiewicz semiring

\section{Introduction}

Semirings play an important role in both algebra and applications. They share several important properties of rings, but, on the other hand, every distributive lattice with the least element can be recognized as an idempotent semiring. Hence, if the addition operation of a semiring is idempotent, then the semiring often shares some properties with semilattices or lattices. Contrary to rings, ideals of semirings need not be zero-classes of congruences, so-called congruence kernels. However, the set of all ideals forms a complete lattice similarly as for rings. We study under what conditions this

Communicated by A. Di Nola.

Support of the research of both authors by ÖAD, Project CZ 04/2017, and by IGA, Project PřF 2018 012, is gratefully acknowledged. Support of the research of the second author by the Austrian Science Fund (FWF), Project I 1923-N25, is gratefully acknowledged.

$凶$ Helmut Länger

helmut.laenger@tuwien.ac.at

Ivan Chajda

ivan.chajda@upol.cz

1 Department of Algebra and Geometry, Faculty of Science, Palacký University Olomouc, 17. listopadu 12, 77146 Olomouc, Czech Republic

2 Faculty of Mathematics and Geoinformation, Institute of Discrete Mathematics and Geometry, TU Wien, Wiedner Hauptstraße 8-10, 1040 Vienna, Austria lattice is complemented. It turns out that for commutative semirings having no 2-nilpotent element, one complement of a given ideal is given by its annihilator. Analogous results are obtained for the lattice of annihilator ideals though this lattice need not be a sublattice of the lattice of ideals.

In the second part we study certain semirings with involution, so-called Łukasiewicz semirings and their ideals. These semirings originated in the study of quantum structures, see, for example, Bonzio et al. (2016) and Chajda et al. (2018) for the concepts and motivation. The ideals of Łukasiewicz semirings have interesting properties, and the congruence kernels of these semirings can be described easily. Complements within the lattice of ideals can be described by means of complemented elements.

It might be the case that commutative semirings are complemented but their complements do not coincide with the corresponding annihilators. We show that by forming the direct product of such semiring with a finite unitary Boolean ring we obtain a commutative semiring whose lattice of ideals has similar properties as the lattice of ideals of the original semiring. Here we use the fact that in such a case we have no skew ideals in the direct product and hence the lattice of ideals of the new semiring is the direct product of the lattices of ideals of its factors. 


\section{Basic concepts}

In the literature there exist different definitions of a semiring. We will use the following one which differs from that in Golan (1999) (where it is called a "hemiring").

A commutative semiring is an algebra $\mathbf{R}=(R,+, \cdot, 0)$ of type $(2,2,0)$ such that

- $(R,+, 0)$ is a commutative monoid,

- $(R, \cdot)$ is a commutative semigroup,

- $(x+y) \cdot z \approx x \cdot z+y \cdot z$,

- $0 \cdot x \approx 0$.

If there exists an element 1 of $R$ satisfying the identity $x \cdot 1 \approx$ $x$ then $\mathbf{R}$ is called unitary, and if $\mathbf{R}$ satisfies the identity $x+x \approx x$ then $\mathbf{R}$ is called idempotent.

For subsets $A, B$ of $R$ put

$$
\begin{aligned}
A+B & :=\{x+y \mid x \in A, y \in B\}, \\
A B & :=\{x \cdot y \mid x \in A, y \in B\} .
\end{aligned}
$$

For $a \in R$ we write $a A$ instead of $\{a\} A$ etc.

An ideal of $\mathbf{R}$ is a subset $I$ of $R$ satisfying $0 \in I, I+I \subseteq I$ and $I R \subseteq I$. Let Id $\mathbf{R}$ denote the set of all ideals of $\mathbf{R}$. Since Id $\mathbf{R}$ is closed with respect to arbitrary set-theoretical intersections, Id $\mathbf{R}:=(\operatorname{Id} R, \subseteq)$ is a complete lattice with the least element $\{0\}$ and the greatest element $R$. Obviously, the lattice operations are given by + and $\cap$. More generally, in Id $\mathbf{R}$ we have

$$
\begin{aligned}
& \bigvee_{s \in S} I_{S}=\sum_{s \in S} I_{S}, \\
& \bigwedge_{s \in S} I_{S}=\bigcap_{s \in S} I_{S}
\end{aligned}
$$

where $\sum_{s \in S} I_{S}$ denotes the set of all sums of finitely many elements of $\bigcup_{s \in S} I_{s}$.

In the following we will write $x y$ instead of $x \cdot y$ and we will denote the set of all nonnegative integers by $\mathbb{N}_{0}$.

Example 2.1 Every commutative ring is a commutative semiring. $\mathbf{N}_{0}:=\left(\mathbb{N}_{0},+, \cdot, 0\right)$ is a commutative semiring which is not a ring since 1 has no additive inverse. Further, $2 \mathbb{N}_{0} \in$ Id $\mathbf{N}_{0}$. Every distributive lattice with 0 is a commutative semiring which is a ring only if it is a singleton. If $\mathbf{L}_{1}=$ $\left(L_{1}, \vee, \wedge, 0\right)$ and $\mathbf{L}_{2}=\left(L_{2}, \vee, \wedge, 0\right)$ are distributive lattices with 0 then $\{0\} \times L_{2} \in \operatorname{Id}\left(\mathbf{L}_{1} \times \mathbf{L}_{2}\right)$.

In order to study the structure of lattices of ideals, we remember several concepts from lattice theory.

Recall that a lattice $(L, \vee, \wedge)$ is called modular if it satisfies the identity

$(x \vee y) \wedge(x \vee z) \approx x \vee(y \wedge(x \vee z))$

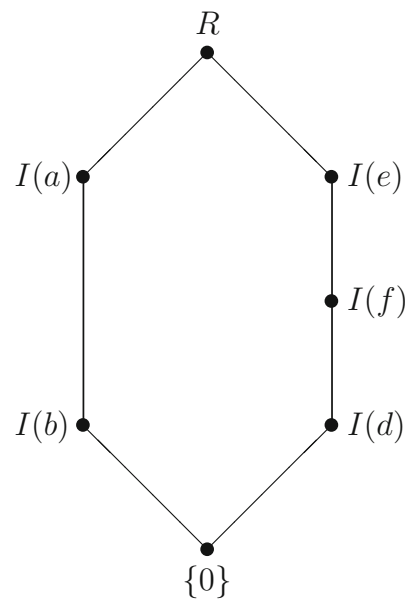

Fig. 1 The lattice of ideals of $\mathbf{R}$ from Example 2.2

It is well known that lattices of ideals of rings are modular. Unfortunately, this need not hold for lattices of ideals of semirings, as the following example shows.

Example 2.2 Let $\mathbf{R}=(R,+, \cdot, 0)$ denote the commutative semiring defined by $R:=\{0, a, b, c, d, e, f, g\}$,

\begin{tabular}{c|llllllll}
+ & 0 & $a$ & $b$ & $c$ & $d$ & $e$ & $f$ & $g$ \\
\hline 0 & 0 & $a$ & $b$ & $c$ & $d$ & $e$ & $f$ & $g$ \\
$a$ & $a$ & $b$ & $c$ & 0 & $e$ & $f$ & $g$ & $d$ \\
$b$ & $b$ & $c$ & 0 & $a$ & $f$ & $g$ & $d$ & $e$ \\
$c$ & $c$ & 0 & $a$ & $b$ & $g$ & $d$ & $e$ & $f$ \\
$d$ & $d$ & $e$ & $f$ & $g$ & $d$ & $e$ & $f$ & $g$ \\
$e$ & $e$ & $f$ & $g$ & $d$ & $e$ & $f$ & $g$ & $d$ \\
$f$ & $f$ & $g$ & $d$ & $e$ & $f$ & $g$ & $d$ & $e$ \\
$g$ & $g$ & $d$ & $e$ & $f$ & $g$ & $d$ & $e$ & $f$
\end{tabular}

and $x y:=0$ for all $x, y \in R$. Evidently, $\mathbf{R}$ is not a ring. $\mathbf{R}$ has the ideals $\{0\}, I(a), I(b), I(d), I(e), I(f), R$ where $I(a):=\{0, a, b, c\}, I(b):=\{0, b\}, I(d):=\{0, d\}, I(e):=$ $\{0, d, e, f, g\}$ and $I(f):=\{0, d, f\}$. The Hasse diagram of Id $\mathbf{R}$ is shown in Fig. 1.

Obviously, for an arbitrary subset $A$ of $R$ the set

$$
\begin{aligned}
I(A) & :=\left\{a_{1} r_{1}+\cdots+a_{k} r_{k}+n_{1} a_{k+1}+\cdots+n_{l} a_{k+l} \mid k, l\right. \\
& \left.\geq 0, a_{1}, \ldots, a_{k+l} \in A, r_{1}, \ldots, r_{k} \in R, n_{1}, \ldots, n_{l} \in \mathbb{N}_{0}\right\}
\end{aligned}
$$

is the ideal generated by $A$. Here, for arbitrary $a \in R$ we have

$0 a:=0,1 a:=a, 2 a:=a+a, \ldots$

Particularly, for $a \in R$ the set

$I(a):=a R+\mathbb{N}_{0} a$ 
(where $\mathbb{N}_{0} a$ denotes the set $\left\{n a \mid n \in \mathbb{N}_{0}\right\}$ ) is the ideal generated by $a$. Such ideals are called principal.

\section{Annihilators as complements of ideals}

For any $I \in \operatorname{Id} \mathbf{R}$ we define $I^{*}:=\{x \in R \mid x I=\{0\}\}$, call $I^{*}$ an annihilator ideal of $\mathbf{R}$ and * annihilation or annihilation mapping and put Ann $\mathbf{R}:=\left\{I^{*} \mid I \in \operatorname{Id} \mathbf{R}\right\}$. It is easy to see that Ann $\mathbf{R} \subseteq \operatorname{Id} \mathbf{R}$. Moreover, Ann $\mathbf{R}:=(\operatorname{Ann} \mathbf{R}, \subseteq)$ is a complete lattice. This follows from (i) of Lemma 3.3.

Example 3.1 Referring to Example 2.1 we have $\left(2 \mathbb{N}_{0}\right)^{*}=$ $\{0\}$ and $\left(\{0\} \times L_{2}\right)^{*}=L_{1} \times\{0\}$ provided $L_{2}$ is not a singleton.

The following facts are straightforward.

Lemma 3.2 Let $\mathbf{R}=(R,+, \cdot, 0)$ be a commutative semiring and $I, J \in \operatorname{Id} \mathbf{R}$. Then

- $I \subseteq J$ implies $J^{*} \subseteq I^{*}$,

- $I \subseteq I^{* *}$,

- $I^{* * *}=I^{*}$,

- $\{0\}^{*}=R$,

- if $\mathbf{R}$ is unitary then $R^{*}=\{0\}$.

From Lemma 3.2 we obtain Ann $\mathbf{R}=\left\{I \in \operatorname{Id} \mathbf{R} \mid I^{* *}=\right.$ I\}.

In the following we are going to investigate the lattice operations in Id R and Ann R. Since these lattices are complete, we consider, more generally, infinite joins and meets.

Lemma 3.3 Let $\mathbf{R}=(R,+, \cdot, 0)$ be a commutative semiring. In $\mathbf{I d} \mathbf{R}$ we have

(i) $\left(\sum_{s \in S} I_{s}\right)^{*}=\bigcap_{s \in S} I_{s}^{*}$,

(ii) $\left(\bigcap_{s \in S} I_{S}\right)^{*} \supseteq \sum_{s \in S} I_{s}^{*}$.

Proof (i) We have $I_{t} \subseteq \sum_{s \in S} I_{s}$ for all $t \in S$ and hence $\left(\sum_{s \in S} I_{S}\right)^{*} \subseteq I_{t}^{*}$ for all $t \in S$ whence $\left(\sum_{s \in S} I_{S}\right)^{*} \subseteq$ $\bigcap_{s \in S} I_{s}^{*}$. On the other hand, if $a \in \bigcap_{s \in S} I_{s}^{*}$ and $b \in$ $\sum_{s \in S} I_{S}$ then there exist $b_{1}, \ldots, b_{n} \in \bigcup_{s \in S} I_{s}$ with $b_{1}+$ $\cdots+b_{n}=b$ and hence

$$
\begin{aligned}
a b & =a\left(b_{1}+\cdots+b_{n}\right)=a b_{1}+\cdots+a b_{n} \\
& =0+\cdots+0=0,
\end{aligned}
$$

i.e. $a \in\left(\sum_{s \in S} I_{S}\right)^{*}$ showing $\bigcap_{s \in S} I_{s}^{*} \subseteq\left(\sum_{s \in S} I_{S}\right)^{*}$ and hence (i).

(ii) We have $\bigcap_{s \in S} I_{s} \subseteq I_{t}$ for all $t \in S$ and hence $I_{t}^{*} \subseteq$ $\left(\bigcap_{s \in S} I_{S}\right)^{*}$ for all $t \in S$ which shows (ii).
As remarked above, since in Ann $\mathbf{R}$ we have

$$
\bigcap_{s \in S} I_{s}=\bigcap_{s \in S} I_{s}^{* *}=\left(\sum_{s \in S} I_{s}^{*}\right)^{*} \in \operatorname{Ann} \mathbf{R},
$$

Ann $\mathbf{R}$ is a complete lattice.

The following lemma shows that in general the supremum within Ann R may differ from that within Id R.

Lemma 3.4 Let $\mathbf{R}=(R,+, \cdot, 0)$ be a commutative semiring. In Ann $\mathbf{R}$ we have

$$
\begin{aligned}
& \bigvee_{s \in S} I_{S}=\left(\sum_{s \in S} I_{S}\right)^{* *}, \\
& \bigwedge_{s \in S} I_{S}=\bigcap_{s \in S} I_{S} .
\end{aligned}
$$

Proof For all $t \in S$ we have $I_{t} \subseteq \sum_{s \in S} I_{S}$ and hence $\left(\sum_{s \in S} I_{S}\right)^{*} \subseteq I_{t}^{*}$ whence $I_{t}=I_{t}^{* *} \subseteq\left(\sum_{s \in S} I_{S}\right)^{* *}$. Moreover, if $I \in$ Ann $\mathbf{R}$ and $I_{s} \subseteq I$ for all $s \in S$ then $\sum_{s \in S} I_{s} \subseteq I$ and hence $I^{*} \subseteq\left(\sum_{s \in S} I_{S}\right)^{*}$ whence $\left(\sum_{s \in S} I_{S}\right)^{* *} \subseteq I^{* *}=$ $I$. This proves (2). Finally, according to (1), Ann $\mathbf{R}$ is closed under arbitrary intersections which yields (3).

The lattice of ideals and the annihilator lattice of $\mathbf{R}$ can be written in the form

$$
\mathbf{I d} \mathbf{R}=(\operatorname{Id} \mathbf{R},+, \cap,\{0\}, R),
$$

Ann $\mathbf{R}=\left(\right.$ Ann $\left.\mathbf{R}, \vee, \cap, R^{*}, R\right)$,

respectively, where

$I \vee J:=(I+J)^{* *}$

for all $I, J \in$ Ann $\mathbf{R}$.

If for an arbitrary subset $A$ of $R$ we define $A^{*}:=\{x \in$ $R \mid x A=\{0\}\}$, then $A^{*}=I(A)^{*}$ and hence Ann $\mathbf{R}=\left\{A^{*} \mid\right.$ $A \subseteq R\}$.

Now, we define the concept which plays a crucial role for complementation in Id R.

Definition 3.5 We call an element $a$ of a commutative semiring $\mathbf{R}=(R,+, \cdot, 0)$ 2-nilpotent if $a \neq 0=a^{2}$.

Recall that an ortholattice (see Birkhoff 1979) is an algebra $\left(L, \vee, \wedge,,^{\prime}, 0,1\right)$ of type $(2,2,1,0,0)$ such that $(L, \vee, \wedge, 0,1)$ is a bounded lattice and the following identities are satisfied:

$$
\begin{gathered}
x \vee x^{\prime} \approx 1, x \wedge x^{\prime} \approx 0,\left(x^{\prime}\right)^{\prime} \approx x,(x \vee y)^{\prime} \approx x^{\prime} \wedge y^{\prime}, \\
(x \wedge y)^{\prime} \approx x^{\prime} \vee y^{\prime} .
\end{gathered}
$$

Hence, in every ortholattice we have $x \leq y$ if and only if $y^{\prime} \leq x^{\prime}$. Such a complementation is called an orthocomplementation, see Birkhoff (1979). 
In the following we set

$\mathbf{I d}^{*} \mathbf{R}:=\left(\operatorname{Id} \mathbf{R},+, \cap,{ }^{*},\{0\}, R\right)$ and

$\mathbf{A n n}^{*} \mathbf{R}:=\left(\operatorname{Ann} \mathbf{R}, \vee, \cap,^{*}, R^{*}, R\right)$

and investigate under which conditions these algebras are ortholattices. Obviously,

$\mathbf{I d}^{*} \mathbf{R}=$ Ann $^{*} \mathbf{R}$ if and only it $I^{* *}=I$ for all $I \in \operatorname{Id} \mathbf{R}$.

Lemma 3.6 Let $\mathbf{R}=(R,+, \cdot, 0)$ be a commutative semiring. Then for every ideal I of $\mathbf{R}$ its pseudocomplement in $\mathbf{I d} \mathbf{R}$ is just $I^{*}$ if and only if $\mathbf{R}$ has no 2-nilpotent element.

Proof First assume $I^{*}$ to be the pseudocomplement of $I$ in Id $\mathbf{R}$ for each $I \in \operatorname{Id} \mathbf{R}$. If $a \in R$ and $a^{2}=0$ then $a \in$ $I(a) \cap I(a)^{*}=\{0\}$ and hence $a=0$. Conversely, assume $\mathbf{R}$ to have no 2-nilpotent element. Let $J, K \in \operatorname{Id} \mathbf{R}$. If $b \in J \cap J^{*}$ then $b^{2}=0$ whence $b=0$ which shows $J \cap J^{*}=\{0\}$. Conversely, if $J \cap K=\{0\}$ then $j k \in J \cap K=\{0\}$ for all $j \in J$ and $k \in K$ and hence $K \subseteq J^{*}$. This shows that $J^{*}$ is the pseudocomplement of $J$ in Id $\mathbf{R}$ completing the proof of the lemma.

Theorem 3.7 Let $\mathbf{R}=(R,+, \cdot, 0)$ be a commutative semiring. Then $\mathbf{I d}^{*} \mathbf{R}$ is an ortholattice if and only if $I^{* *}=I$ for all $I \in \mathrm{Id} \mathbf{R}$ and $\mathbf{R}$ has no 2-nilpotent element.

Proof First assume $\mathbf{I d}^{*} \mathbf{R}$ to be an ortholattice. Then $I^{* *}=I$ for all $I \in \operatorname{Id} \mathbf{R}$. Moreover, if $a \in R$ and $a^{2}=0$ then $a \in I(a) \cap I(a)^{*}=\{0\}$ and hence $a=0$. Conversely, assume $I^{* *}=I$ for all $I \in \operatorname{Id} \mathbf{R}$ and $\mathbf{R}$ to have no 2-nilpotent element. Let $J \in \operatorname{Id} \mathbf{R}$. According to Lemma 3.6, $J \cap J^{*}=$ $\{0\}$. Now, according to (i) of Lemma 3.3,

$J+J^{*}=\left(J+J^{*}\right)^{* *}=\left(J^{*} \cap J^{* *}\right)^{*}=\left(J^{*} \cap J\right)^{*}=\{0\}^{*}=R$.

Finally, the de Morgan laws hold because of Lemma 3.2.

The following two examples show which role the existence of 2-nilpotent elements plays for the fact of annihilators to be complements.

Example 3.8 Let $\mathbf{R}=(R,+, \cdot, 0)$ denote the commutative semiring with $R:=\{0, a, b, c, d, e\}$ and

\begin{tabular}{c|lllllll|llllll}
+ & 0 & $a$ & $b$ & $c$ & $d$ & $e$ & $\cdot$ & 0 & $a$ & $b$ & $c$ & $d$ & $e$ \\
\hline 0 & 0 & $a$ & $b$ & $c$ & $d$ & $e$ & 0 & 0 & 0 & 0 & 0 & 0 & 0 \\
$a$ & $a$ & $b$ & 0 & $d$ & $e$ & $c$ & $a$ & 0 & $a$ & $b$ & 0 & $a$ & $b$ \\
$b$ & $b$ & 0 & $a$ & $e$ & $c$ & $d$ & $b$ & 0 & $b$ & $a$ & 0 & $b$ & $a$ \\
$c$ & $c$ & $d$ & $e$ & $c$ & $d$ & $e$ & $c$ & 0 & 0 & 0 & $c$ & $c$ & $c$ \\
$d$ & $d$ & $e$ & $c$ & $d$ & $e$ & $c$ & $d$ & 0 & $a$ & $b$ & $c$ & $d$ & $e$ \\
$e$ & $e$ & $c$ & $d$ & $e$ & $c$ & $d$ & $e$ & 0 & $b$ & $a$ & $c$ & $e$ & $d$
\end{tabular}

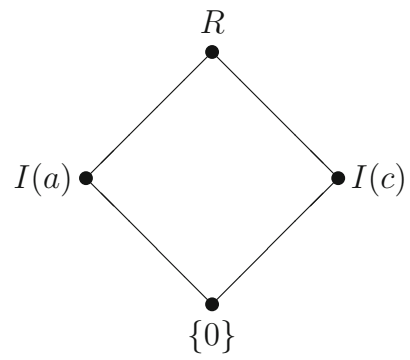

Fig. 2 The lattice of ideals of $\mathbf{R}$ from Example 3.8

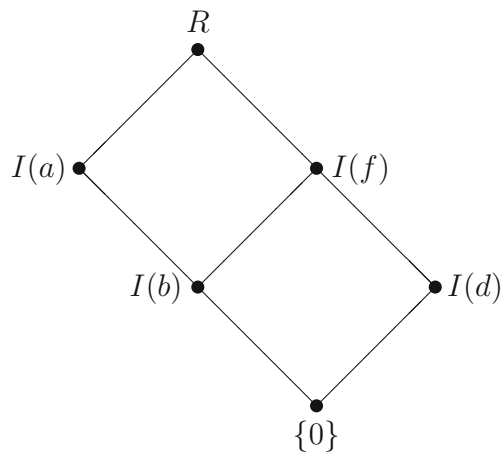

Fig. 3 The lattice of ideals of $\mathbf{R}$ from Example 3.9

Then $\mathbf{R}$ is not a ring. Its ideals are $\{0\}, I(a), I(c)$ and $R$ where $I(a)=\{0, a, b\}$ and $I(c)=\{0, c\}$. It is easy to check that $\{0\}^{*}=R, I(a)^{*}=I(c), I(c)^{*}=I(a)$ and $R^{*}=\{0\}$. Moreover, $\mathbf{R}$ does not contain a 2-nilpotent element. Thus, for each ideal $I$ of $\mathbf{R}, I^{*}$ is a complement of $I$ in $\mathbf{I d} \mathbf{R}$. The Hasse diagram of Id $\mathbf{R}$ is depicted in Fig. 2.

Example 3.9 Let $\mathbf{R}=(R,+, \cdot, 0)$ denote the commutative semiring with $R:=\{0, a, b, c, d, e, f, g\}$,

\begin{tabular}{c|lllllllll|llllllll}
+ & 0 & $a$ & $b$ & $c$ & $d$ & $e$ & $f$ & $g$ & $\cdot$ & 0 & $a$ & $b$ & $c$ & $d$ & $e$ & $f$ & $g$ \\
\hline 0 & 0 & $a$ & $b$ & $c$ & $d$ & $e$ & $f$ & $g$ & 0 & 0 & 0 & 0 & 0 & 0 & 0 & 0 & 0 \\
$a$ & $a$ & $b$ & $c$ & 0 & $e$ & $f$ & $g$ & $d$ & $a$ & 0 & $a$ & $b$ & $c$ & 0 & $a$ & $b$ & $c$ \\
$b$ & $b$ & $c$ & 0 & $a$ & $f$ & $g$ & $d$ & $e$ & $b$ & 0 & $b$ & 0 & $b$ & 0 & $b$ & 0 & $b$ \\
$c$ & $c$ & 0 & $a$ & $b$ & $g$ & $d$ & $e$ & $f$ & $c$ & 0 & $c$ & $b$ & $a$ & 0 & $c$ & $b$ & $a$ \\
$d$ & $d$ & $e$ & $f$ & $g$ & $d$ & $e$ & $f$ & $g$ & $d$ & 0 & 0 & 0 & 0 & $d$ & $d$ & $d$ & $d$ \\
$e$ & $e$ & $f$ & $g$ & $d$ & $e$ & $f$ & $g$ & $d$ & $e$ & 0 & $a$ & $b$ & $c$ & $d$ & $e$ & $f$ & $g$ \\
$f$ & $f$ & $g$ & $d$ & $e$ & $f$ & $g$ & $d$ & $e$ & $f$ & 0 & $b$ & 0 & $b$ & $d$ & $f$ & $d$ & $f$ \\
$g$ & $g$ & $d$ & $e$ & $f$ & $g$ & $d$ & $e$ & $f$ & $g$ & 0 & $c$ & $b$ & $a$ & $d$ & $g$ & $f$ & $e$
\end{tabular}

It is easy to check that $\mathbf{R}$ is not a ring. $\mathbf{R}$ has the following ideals: $\{0\}, I(a), I(b), I(d), I(f)$ and $R$ where $I(a)=\{0, a, b, c\}, I(b)=\{0, b\}, I(d)=\{0, d\}$ and $I(f)=\{0, b, d, f\}$. The Hasse diagram of Id $\mathbf{R}$ is visualized in Fig. 3.

The element $b$ is 2-nilpotent and hence, according to Theorem 3.7, $\mathbf{I} \mathbf{d}^{*} \mathbf{R}$ is not an ortholattice as can be seen from $I(b) \vee I(b)^{*}=I(b) \vee I(f)=I(f) \neq R$. 
It is easy to see that $\{0\}^{* *} \neq\{0\}$ if and only if there exists some $x \in R \backslash\{0\}$ with $x R=\{0\}$. Hence, if there exists such an element $x$, then $\mathbf{I} \mathbf{d}^{*} \mathbf{R}$ is not an ortholattice.

If $\mathbf{A n n}^{*} \mathbf{R}$ is considered instead of $\mathbf{I d}^{*} \mathbf{R}$, then the condition for being an ortholattice can be simplified as follows:

Theorem 3.10 Let $\mathbf{R}=(R,+, \cdot, 0)$ be a commutative semiring. Then Ann* $\mathbf{R}$ is an ortholattice if and only if there exists no $a \in R \backslash R^{*}$ with $a^{2}=0$.

Proof First assume Ann* $\mathbf{R}$ to be an ortholattice. If $a \in R$ and $a^{2}=0$ then $a \in I(a)^{*} \cap I(a)^{* *}=R^{*}$. Conversely, assume that there exists no $b \in R \backslash R^{*}$ with $b^{2}=0$. Let $J \in$ Ann R. If $c \in J \cap J^{*}$ then $c^{2}=0$ whence $c \in R^{*}$ which shows $J \cap J^{*}=R^{*}$. Finally, according to (i) of Lemma 3.3,

$J \vee J^{*}=\left(J+J^{*}\right)^{* *}=\left(J^{*} \cap J^{* *}\right)^{*}=\left(J^{*} \cap J\right)^{*}=R^{* *}=R$

completing the proof of the theorem.

It is natural to ask under which condition ${ }^{* *}$ is a homomorphism from Id* $\mathbf{R}$ onto Ann* $\mathbf{R}$. A sufficient condition is presented by the following theorem.

Theorem 3.11 Let $\mathbf{R}=(R,+, \cdot, 0)$ be a commutative semiring and assume

$(I \cap J)^{* *}=I^{* *} \cap J^{* *}$ for all $I, J \in \mathrm{Id} \mathbf{R}$.

Then $^{* *}$ is a homomorphism from $\mathbf{I d}^{*} \mathbf{R}$ onto Ann ${ }^{*} \mathbf{R}$. Moreover, we have

$$
\begin{aligned}
& \left(\sum_{s \in S} I_{S}\right)^{* *}=\bigvee I_{s}^{* *} \text { and } \\
& \left(\bigcap_{s \in S} I_{S}\right)^{* *} \subseteq \bigcap_{s \in S} I_{s}^{* *}
\end{aligned}
$$

for every family $\left(I_{S} ; s \in S\right)$ of ideals of $\mathbf{R}$.

Proof Let $I, J \in \operatorname{Id} \mathbf{R}$. Then

$$
\begin{aligned}
(I+J)^{* *} & =\left(I^{*} \cap J^{*}\right)^{*}=\left(I^{* * *} \cap J^{* * *}\right)^{*} \\
& =\left(I^{* *}+J^{* *}\right)^{* *}=I^{* *} \vee J^{* *}, \\
(I \cap J)^{* *} & =I^{* *} \cap J^{* *}, \\
\left(I^{*}\right)^{* *} & =\left(I^{* *}\right)^{*}, \\
\{0\}^{* *} & =R^{*} \text { and } \\
R^{* *} & =R .
\end{aligned}
$$

Moreover, we have

$$
\left(\sum_{s \in S} I_{S}\right)^{* *}=\left(\bigcap_{s \in S} I_{s}^{*}\right)^{*}=\left(\bigcap I_{s}^{* * *}\right)^{*}
$$

$$
=\left(\sum_{s \in S} I_{s}^{* *}\right)^{* *}=\bigvee_{s \in S} I_{s}^{* *}
$$

for every family $\left(I_{s} ; s \in S\right.$ ) of ideals of $\mathbf{R}$. The rest of the proof is clear.

For any commutative semiring $\mathbf{R}$ and any $\Theta \in$ Con $\mathbf{R}$ we call $[0] \Theta$ the kernel of $\Theta$.

In contrast to the case of commutative rings, an ideal of a commutative semiring $\mathbf{R}$ need not be the kernel of some congruence on $\mathbf{R}$. We have only $[0] \Theta \in$ Id $\mathbf{R}$ provided $\Theta \in$ Con R. A weaker result holds for the so-called Bourne congruence induced by an ideal $I$ of $\mathbf{R}$ :

Theorem 3.12 Let I be some ideal of a commutative semiring $\mathbf{R}=(R,+, \cdot, 0)$ and put

$\Theta(I):=\left\{(x, y) \in R^{2} \mid\right.$ there exist $a, b \in I$ with $\left.x+a=y+b\right\}$.

The $\Theta(I) \in$ Con $\mathbf{R}$ and $I \subseteq[0] \Theta(I)$.

The proof is easy (cf. Proposition 6.50 in Golan 1999) and therefore omitted.

\section{Ideals and congruence kernels in Łukasiewicz semirings}

In the following we investigate commutative semirings with a unary operation ' . For such extended semirings we study complements in the lattice of ideals which are not derived by annihilators as above, but by means of this unary operation.

The concept of a Łukasiewicz semiring was introduced in Bonzio et al. (2016) and Chajda et al. (2018) as an algebraic semantic of a certain non-classical logic used in quantum mechanics. The motivation can be found in Chajda et al. (2018) and Bonzio et al. (2016).

Namely, within the logic of quantum mechanics effects are described by means of the so-called effect algebras. Z. Riečanová proved that every lattice effect algebra is built up by blocks which are in fact MV-algebras. MV-algebras were introduced in the 1950's by C. C. Chang as an algebraic axiomatization of Łukasiewicz many-valued logics. As shown in Chajda et al. (2018), MV-algebras are in oneto-one correspondence with Łukasiewicz semirings. Using these semirings instead of MV-algebras, one can apply the theory of semirings developed, e.g. in Golan (1999), i.e. the theory of ideals and annihilators in particular.

A Eukasiewicz semiring is an algebra $\mathbf{R}=\left(R,+, \cdot,{ }^{\prime}, 0,1\right)$ of type $(2,2,1,0,0)$ such that

(i) $(R,+, \cdot, 0,1)$ is a unitary and idempotent commutative semiring, 
(ii) $x+1 \approx 1$

(iii) $\left(x^{\prime} y\right)^{\prime} y \approx\left(y^{\prime} x\right)^{\prime} x$

(iv) ' is an antitone involution of the poset $(R, \leq)$ where $\leq$ denotes the partial order relation corresponding to the bounded join-semilattice $(R,+, 0,1)$.

The special case $y=0$ in (iii) yields $x x^{\prime} \approx 0$. As shown in Chajda et al. (2018), the following identity holds in every Łukasiewicz semiring:

$x+y \approx\left(\left(x y^{\prime}\right)^{\prime} y^{\prime}\right)^{\prime}$.

If $a \in R$ satisfies $a^{2}=a$, then the previous identity yields

$a+a^{\prime}=\left((a a)^{\prime} a\right)^{\prime}=\left(a^{\prime} a\right)^{\prime}=0^{\prime}=1$.

Since Łukasiewicz semirings are commutative semirings, we use the same definition of an ideal as before.

Lemma 4.1 Let $\mathbf{R}=\left(R,+, \cdot,{ }^{\prime}, 0,1\right)$ be a Łukasiewicz semiring and $I$ a subset of $R$ with $0 \in I$ satisfying

If $x \in R$ and $y, x y^{\prime} \in I$, then $x \in I$.

Then $I \in \operatorname{Id} \mathbf{R}$.

Proof Let $a, b \in I$ and $c \in R$. We have $\left(a^{\prime} b\right) b^{\prime}=a^{\prime} b b^{\prime}=$ $0 \in I$ whence $a^{\prime} b \in I$ according to (5). Moreover, $(a+$ b) $a^{\prime}=a a^{\prime}+b a^{\prime}=a^{\prime} b \in I$ whence $a+b \in I$ again according to (5). Finally, $(a c) a^{\prime}=a a^{\prime} c=0 \in I$ whence $a c \in I$ according to (5) completing the proof of the lemma.

For every Łukasiewicz semiring $\mathbf{R}=\left(R,+, \cdot,^{\prime}, 0,1\right)$ let $\mathrm{CK}(\mathbf{R})$ denote the set of all subsets $I$ of $R$ with $0 \in I$ satisfying (5) and the following condition:

$x, y, z \in R$ and $x y^{\prime} \in I$ imply $x z(y z)^{\prime} \in I$.

According to Lemma 4.1, $\mathrm{CK}(\mathbf{R}) \subseteq \operatorname{Id} \mathbf{R}$. Put CK R := $(\mathrm{CK} \mathbf{R}, \subseteq)$.

Lemma 4.2 Let $\mathbf{R}=\left(R,+, \cdot,{ }^{\prime}, 0,1\right)$ be a Eukasiewicz semiring and $\Theta \in$ Con $\mathbf{R}$. Then $[0] \Theta \in$ CK R.

Proof Let $a, b, c \in R$. Obviously, $0 \in[0] \Theta$. If $b, a b^{\prime} \in[0] \Theta$ then $a=a 1=a 0^{\prime} \in\left[a b^{\prime}\right] \Theta=[0] \Theta$. Finally, if $a b^{\prime} \in[0] \Theta$ then

$$
\begin{aligned}
a c(b c)^{\prime} & =1 a c(b c)^{\prime}=0^{\prime} a c(b c)^{\prime} \in\left[\left(b^{\prime} a\right)^{\prime} a c(b c)^{\prime}\right] \Theta \\
& =\left[\left(a^{\prime} b\right)^{\prime} b c(b c)^{\prime}\right] \Theta=[0] \Theta .
\end{aligned}
$$

Next we prove that CK $\mathbf{R}$ is just the set of congruence kernels of $\mathbf{R}$.
Theorem 4.3 Let $\mathbf{R}=\left(R,+, \cdot,{ }^{\prime}, 0,1\right)$ be a Eukasiewicz semiring and $I \in \mathrm{CK} \mathbf{R}$. Put

$\Theta(I):=\left\{(x, y) \in R^{2} \mid x y^{\prime}, x^{\prime} y \in I\right\}$.

Then $\Theta(I) \in$ Con $\mathbf{R}$.

Proof Let $a, b, c \in R$. It is evident that $\Theta(I)$ is reflexive and symmetric. Now assume $(a, b),(b, c) \in \Theta(I)$, i.e. $a b^{\prime}, a^{\prime} b, b c^{\prime}, b^{\prime} c \in I$. Since

$a c^{\prime}\left(b c^{\prime}\right)^{\prime}=a\left(b c^{\prime}\right)^{\prime} c^{\prime}=a\left(c b^{\prime}\right)^{\prime} b^{\prime} \in I$

and

$a^{\prime} c\left(b^{\prime} c\right)^{\prime}=a^{\prime}\left(b^{\prime} c\right)^{\prime} c=a^{\prime}\left(c^{\prime} b\right)^{\prime} b \in I$

we have $a c^{\prime}, a^{\prime} c \in I$ according to (5), i.e. $(a, c) \in \Theta(I)$ showing transitivity of $\Theta(I)$. Next assume $(a, b) \in \Theta(I)$, i.e. $a b^{\prime}, a^{\prime} b \in I$. Then

$a c(b c)^{\prime}=a(b c)^{\prime} c=a\left(c^{\prime} b^{\prime}\right)^{\prime} b^{\prime} \in I$

and

$(a c)^{\prime} b c=(a c)^{\prime} c b=\left(c^{\prime} a^{\prime}\right)^{\prime} a^{\prime} b \in I$

showing $(a c, b c) \in \Theta(I)$ according to (5). Obviously, $\Theta(I)$ is compatible with '. Finally, $(a, b) \in \Theta(I)$ implies $(a+$ $c, b+c)=\left(\left(\left(a c^{\prime}\right)^{\prime} c^{\prime}\right)^{\prime},\left(\left(b c^{\prime}\right)^{\prime} c^{\prime}\right)^{\prime}\right) \in \Theta(I)$ completing the proof of the lemma.

Theorem 4.4 Let $\mathbf{R}=\left(R,+, \cdot,^{\prime}, 0,1\right)$ be a Łukasiewicz semiring. Then $\mathbf{C K} \mathbf{R}$ is isomorphic to $\mathbf{C o n} \mathbf{R}:=($ Con $\mathbf{R}, \subseteq)$ and hence a complete lattice. The infimum in $\mathbf{C K} \mathbf{R}$ coincides with the set-theoretical intersection. Moreover, the correspondence described in Lemma 4.2 and Theorem 4.3 is one-to-one.

Proof Let $a, b \in R, \Phi, \Psi \in$ Con $\mathbf{R}$ and $I, J \in \mathrm{CK} \mathbf{R}$. If $(a, b) \in \Theta([0] \Phi)$ then $a b^{\prime}, a^{\prime} b \in[0] \Phi$ and hence

$a=1 a=0^{\prime} a \Phi\left(b^{\prime} a\right)^{\prime} a=\left(a^{\prime} b\right)^{\prime} b \Phi 0^{\prime} b=1 b=b$.

If, conversely, $(a, b) \in \Phi$ then $a b^{\prime}, a^{\prime} b \in\left[a a^{\prime}\right] \Phi=[0] \Phi$ and hence $(a, b) \in \Theta([0] \Phi)$. This shows $\Theta([0] \Phi)=\Phi$. Moreover, the following are equivalent: $a \in[0] \Theta(I)$; $a 0^{\prime}, a^{\prime} 0 \in I ; a \in I$. This shows $[0] \Theta(I)=I$. Finally, $\Phi \subseteq$ $\Psi$ implies $[0] \Phi \subseteq[0] \Psi$, and $I \subseteq J$ implies $\Theta(I) \subseteq \Theta(J)$. That the infimum in $\mathbf{C K} \mathbf{R}$ coincides with set-theoretical intersection follows from $\bigcap_{i \in I}\left([0] \Theta_{i}\right)=[0]\left(\bigcap_{i \in I} \Theta_{i}\right)$ for every family $\Theta_{i}, i \in I$, of congruences on $\mathbf{R}$. 
Since in a Łukasiewicz semiring $\mathbf{R}=\left(R,+, \cdot{ }^{\prime}, 0,1\right)$ we have $x \vee y=x+y$ and since' ${ }^{\prime}$ is an antitone involution on the poset $(R, \leq)$ corresponding to the join-semilattice $(R,+)$, we can use De Morgan laws and obtain $x \wedge y=\left(x^{\prime}+y^{\prime}\right)^{\prime}$.

The following lemma lists some important properties of Łukasiewicz semirings.

Lemma 4.5 Let $\mathbf{R}=\left(R,+, \cdot,{ }^{\prime}, 0,1\right)$ be a tukasiewicz semiring and $a, b, c \in R$. Then the following hold:

(i) If $a \leq b$ then $a c \leq b c$,

(ii) $a \leq b$ if and only if $a b^{\prime}=0$,

(iii) if $a+b=1$ and $a b=0$ then $b=a^{\prime}$,

(iv) $a b \leq a \wedge b$,

(v) $I(a)=a R$.

Proof (i) If $a \leq b$ then $a+b=b$ and hence $a c+b c=$ $(a+b) c=b c$ whence $a c \leq b c$.

(ii) If $a \leq b$ then $a b^{\prime} \leq b b^{\prime}=0$ according to (i) and hence $a b^{\prime}=0$. Conversely, if $a b^{\prime}=0$ then

$a+b=\left(\left(a b^{\prime}\right)^{\prime} b^{\prime}\right)^{\prime}=\left(0^{\prime} b^{\prime}\right)^{\prime}=\left(1 b^{\prime}\right)^{\prime}=\left(b^{\prime}\right)^{\prime}=b$,

i.e. $a \leq b$.

(iii) If $a+b=1$ and $a b=0$ then $b \leq a^{\prime}$ according to (ii) and

$a^{\prime} b^{\prime}=1 a^{\prime} b^{\prime}=(a+b) a^{\prime} b^{\prime}=a a^{\prime} b+a b b^{\prime}=0+0=0$

whence $b \geq a^{\prime}$ again according to (ii) and hence $b=a^{\prime}$.

(iv) We have

$$
a b=\left(a^{\prime}\right)^{\prime} b=\left(a^{\prime} 1\right)^{\prime} b \leq\left(a^{\prime} b\right)^{\prime} b=\left(a^{\prime}+b^{\prime}\right)^{\prime}=a \wedge b
$$

according to (i).

(v) This follows since $\mathbf{R}$ is unitary.

For any two ideals $I, J$ of a commutative semiring $\mathbf{R}=$ $(R,+, \cdot, 0)$ we have $I J \subseteq I \cap J$, but in general we do not have equality. However, in some cases equality follows.

Lemma 4.6 Let $\mathbf{R}=(R,+, \cdot, 0,1)$ be a unitary commutative semiring and $I, J \in \operatorname{Id} \mathbf{R}$ satisfying $I+J=R$ and $I J=\{0\}$. Then $I J=I \cap J$.

Proof Since $I+J=R$ there exists $a \in I$ and $b \in J$ with $a+b=1$. Now $x=x 1=x(a+b)=x a+x b=0$ for all $x \in I \cap J$ completing the proof of the lemma.

Theorem 4.7 Let $\mathbf{R}=\left(R,+, \cdot,{ }^{\prime}, 0,1\right)$ be a Eukasiewicz semiring and $I, J \in \operatorname{Id} \mathbf{R}$. Then I and $J$ are complements of each other in $\mathbf{I d} \mathbf{R}$ if and only if there exists some $a \in R$ with $a+a^{\prime}=1, I(a)=I$ and $I\left(a^{\prime}\right)=J$.
Proof First assume $I$ and $J$ to be complements of each other in Id R. Since $I+J=R$ there exist $a \in I$ and $b \in J$ with $a+b=1$. Since $I J \subseteq I \cap J=\{0\}$ we have $a b=0$. According to (iii) of Lemma 4.5 we conclude $b=a^{\prime}$. If $c \in I$ then $c a^{\prime} \in I J=\{0\}$, i.e. $c a^{\prime}=0$, and hence $c=$ $c 1=c\left(a+a^{\prime}\right)=c a+c a^{\prime}=c a+0=c a \in I(a)$ proving $I \subseteq I(a)$. Since the converse inclusion is evident, we have $I(a)=I$. If $d \in J$ then $a d \in I J=\{0\}$, i.e. $a d=0$, and hence $d=1 d=\left(a+a^{\prime}\right) d=a d+a^{\prime} d=0+a^{\prime} d=$ $a^{\prime} d \in I\left(a^{\prime}\right)$ proving $J \subseteq I\left(a^{\prime}\right)$. Since the converse inclusion is evident, we have $I\left(a^{\prime}\right)=J$. Conversely, assume $e \in R$, $e+e^{\prime}=1, I(e)=I$ and $I\left(e^{\prime}\right)=J$. Since $e+e^{\prime}=1$, we have $x=x 1=x\left(e+e^{\prime}\right)=x e+x e^{\prime} \in I+J$ for all $x \in R$, i.e. $I+J=R$. Now $I J=R e R e^{\prime}=\{0\}$. According to Lemma 4.6, $I \cap J=\{0\}$ which shows that $I$ and $J$ are complements of each other in Id R.

\section{Direct product of a commutative semiring and a finite unitary Boolean ring}

If $\mathbf{R}_{1}=\left(R_{1},+, \cdot, 0\right)$ and $\mathbf{R}_{2}=\left(R_{2},+, \cdot, 0\right)$ are commutative semirings, $I_{1} \in \operatorname{Id} \mathbf{R}_{1}$ and $I_{2} \in \operatorname{Id} \mathbf{R}_{2}$ then $I_{1} \times I_{2} \in$ $\operatorname{Id}\left(\mathbf{R}_{1} \times \mathbf{R}_{2}\right)$. An ideal of $\mathbf{R}_{1} \times \mathbf{R}_{2}$ which is not of this form is called skew.

If $\mathbf{R}_{1} \times \mathbf{R}_{2}$ has no skew ideals, then $\mathbf{I d}\left(\mathbf{R}_{1} \times \mathbf{R}_{2}\right) \cong \mathbf{I d} \mathbf{R}_{1} \times$ Id $\mathbf{R}_{2}$ and hence $\mathbf{I d}\left(\mathbf{R}_{1} \times \mathbf{R}_{2}\right)$ is complemented if and only if $\mathbf{I} \mathbf{d} \mathbf{R}_{1}$ and $\mathbf{I d} \mathbf{R}_{2}$ have this property. We are going to show that if one of two given commutative semirings is a finite unitary Boolean ring then the lattice of ideals of their direct product turns out to be directly decomposable. Recall that a ring is called Boolean if it satisfies the identity $x^{2} \approx x$. It is well known that such rings are commutative and satisfy the identity $x+x \approx 0$.

Lemma 5.1 Let $\mathbf{R}=(R,+, \cdot, 0)$ be a commutative semiring and $\mathbf{B}=(B,+, \cdot, 0)$ a finite unitary Boolean ring. Then $\mathbf{R} \times \mathbf{B}$ has no skew ideals.

Proof If $|B|=1$ then the assertion of the lemma is trivial. Now assume $|B|=2$. Then $\mathbf{B} \cong \mathbb{Z}_{2}$. Let $I \in \operatorname{Id}\left(\mathbf{R} \times \mathbb{Z}_{2}\right)$. If $I \subseteq R \times\{0\}$ then $I$ is not a skew ideal. Hence, assume $I \nsubseteq R \times\{0\}$. Then there exists some $a \in R$ with $(a, 1) \in$ $I$. We conclude $(0,1)=(a, 1)(0,1) \in I$. Let $b \in R$. If $(b, 0) \in I$ then $(b, 1)=(b, 0)+(0,1) \in I$. If, conversely, $(b, 1) \in I$ then $(b, 0)=(b, 1)+(0,1) \in I$. This shows that $(b, 0) \in I$ if and only if $(b, 1) \in I$. Hence, $I$ is not a skew ideal of $\mathbf{R} \times \mathbb{Z}_{2}$. If, finally, $|B|>2$ then $\mathbf{B}$ is a direct product of $n \geq 2$ copies of $\mathbb{Z}_{2}$ and the assertion follows by induction on $n$.

Corollary 5.2 If $\mathbf{R}$ is a commutative semiring whose lattice of ideals is complemented or modular or distributive and $\mathbf{B}$ 


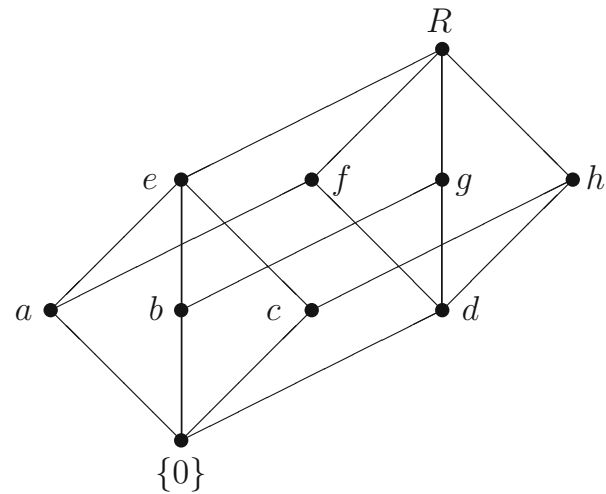

Fig. 4 The lattice of ideals of $\mathbf{R}$ from Example 5.3

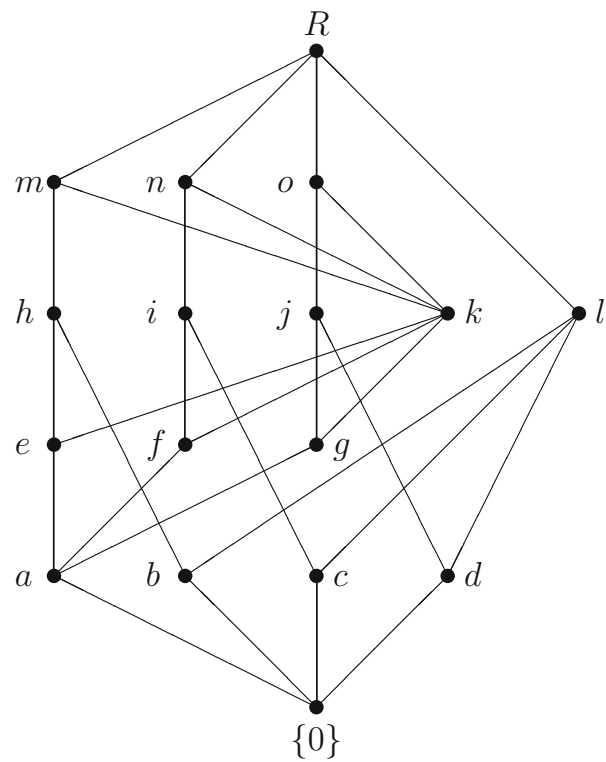

Fig. 5 The lattice of ideals of $\mathbf{R}_{1}$ from Example 5.3

is a finite unitary Boolean ring, then $\mathbf{R} \times \mathbf{B}$ is a commutative semiring whose lattice of ideals is complemented or modular or distributive, respectively.

Hence, if $\mathbf{R}$ denotes the commutative semiring from Example 2.2 whose lattice of ideals is complemented (but complements do not coincide with annihilators in general) and $\mathbf{B}$ is a finite unitary Boolean ring, then $\mathbf{R} \times \mathbf{B}$ is a commutative semiring whose lattice of ideals is complemented, too. Hence, using our procedure, we may produce infinitely many commutative semirings having the mentioned property.

We are going to show that the finite unitary Boolean ring in the previous corollary cannot be substituted by a bounded distributive lattice even in the case when this finite unitary Boolean ring is the two-element one.
Example 5.3 Consider the Kleinian four-element group as the additive group of a zero-ring. Denote this ring by $\mathbf{K}$. Now consider the two-element unitary Boolean ring $\mathbf{B}$ and put $\mathbf{R}:=\mathbf{K} \times \mathbf{B}$. Since $\mathbf{R}$ is a ring, its lattice of ideals is modular (even complemented) and its Hasse diagram is shown in Fig. 4.

The structure of Id $\mathbf{R}$ follows directly from Lemma 5.1 since

$\operatorname{Id} \mathbf{R}=\operatorname{Id}\left(\mathbf{K} \times \mathbf{B}_{2}\right) \cong \operatorname{Id} \mathbf{K} \times \operatorname{Id~} \mathbf{B}_{2} \cong \mathbf{M}_{3} \times \mathbf{2}$.

If, however, we substitute $\mathbf{B}$ by the two-element distributive lattice (considered as a commutative semiring), we obtain a semiring $\mathbf{R}_{1}$ which is not a ring and whose lattice of ideals contains skew ideals. This lattice of ideals is not modular. Its Hasse diagram is depicted in Fig. 5.

However, Id $\mathbf{R}_{1}$ is complemented:

$\{0\}$ is a complement of $R$,

$b$ is a complement of $i, j, n$ and $o$,

$c$ is a complement of $h$,

$d$ is a complement of $m$, and

$l$ is a complement of $a, e, f, g$ and $k$.

Acknowledgements Open access funding provided by Austrian Science Fund (FWF).

Funding This study was funded by ÖAD, Project CZ 04/2017, and by IGA, Project PřF 2018 012, as well as concerning the second author by the Austrian Science Fund (FWF), Project I 1923-N25.

\section{Compliance with ethical standards}

Conflict of interest The authors declare that they have no conflict of interest

Human and animal rights This article does not contain any studies with human participants or animals performed by any of the authors.

Open Access This article is distributed under the terms of the Creative Commons Attribution 4.0 International License (http://creativecomm ons.org/licenses/by/4.0/), which permits unrestricted use, distribution, and reproduction in any medium, provided you give appropriate credit to the original author(s) and the source, provide a link to the Creative Commons license, and indicate if changes were made.

\section{References}

Birkhoff G (1979) Lattice theory. American Mathematical Society, Providence [ISBN 0-8218-1025-1]

Bonzio S, Chajda I, Ledda A (2016) Representing quantum structures as near semirings. Log J IGPL 24:719-742

Chajda I, Fazio D, Ledda A (2018) On the structure theory of Łukasiewicz near semirings. Log J IGPL 26:14-28

Golan JS (1999) Semirings and their applications. Kluwer, Dordrecht [ISBN 0-7923-5786-8]

Publisher's Note Springer Nature remains neutral with regard to jurisdictional claims in published maps and institutional affiliations. 